\title{
Blind Search for 21-cm Absorption Systems in New Generation Chinese Radio Telescopes
}

\author{
Hao-Ran Yu ${ }^{1,2}$, Ue-Li Pen ${ }^{2,3,4,5}$, Tong-Jie Zhang ${ }^{6}$, Di Li ${ }^{7,8}$ and Xuelei Chen ${ }^{9}$ \\ 1 Kavli Institute for Astronomy \& Astrophysics, Peking University, Beijing 100871, China; \\ 2 Canadian Institute for Theoretical Astrophysics, University of Toronto, Toronto, M5S 3H8, ON, \\ Canada; \\ 3 Dunlap Institute for Astronomy and Astrophysics, University of Toronto, Toronto, M5S 3H4, ON, \\ Canada; \\ ${ }^{4}$ Canadian Institute for Advanced Research, Program in Cosmology and Gravitation; \\ 5 Perimeter Institute for Theoretical Physics, Waterloo, ON, N2L 2Y5, Canada; \\ ${ }^{6}$ Department of Astronomy, Beijing Normal University, Beijing, 100875, China; \\ 7 National Astronomical Observatories, Chinese Academy of Sciences, Beijing, China; \\ 8 Key Laboratory of Radio Astronomy, Chinese Academy of Sciences, Beijing, China; \\ ${ }^{9}$ Key Laboratory for Computational Astrophysics, National Astronomical Observatories, Chinese \\ Academy of Sciences, Beijing, 100012, China.
}

\begin{abstract}
Neutral hydrogen clouds are known to exist in the Universe, however their spatial distributions and physical properties are poorly understood. Such missing information can be studied by the new generation Chinese radio telescopes through a blind searching of 21-cm absorption systems. We forecast the capabilities of surveys of $21-\mathrm{cm}$ absorption systems by two representative radio telescopes in China - Five-hundred-meter Aperture Spherical radio Telescope (FAST) and Tianlai $21-\mathrm{cm}$ cosmology experiment (Tianlai). Facilitated by either the high sensitivity (FAST) or the wide field of view (Tianlai) of these telescopes, more than a thousand 21-cm absorption systems can be discovered in a few years, representing orders of magnitude improvement over the cumulative discoveries in the past half a century.
\end{abstract}

\section{Key words:}

\section{INTRODUCTION}

Neutral hydrogen (HI) clouds are known to exist in the Universe, however relatively few of them are discovered in the past half a century, and we poorly understand their spatial distribution and physical properties (Wolfe et al. 2005). In damped Lyman- $\alpha$ absorption (DLA) systems, the radio spectrum is also substantially absorbed by the $\mathrm{HI}$ hyperfine structure, whose rest frame wavelength is approximately $21 \mathrm{~cm}\left(1420405751.7667 \pm 0.009 \mathrm{~Hz}\right.$ in frequency). These systems have at least $2 \times 10^{20} \mathrm{~cm}^{-2} \mathrm{HI}$ column density, thus being able to absorb the background with its cold $\mathrm{HI}$ in their cold neutral medium (CNM).

The 21-cm absorption systems are important in the study of the distribution, location, temperature and structure of neutral gas, and the evolution of neutral gas systems and galaxies over cosmic time scale. Due to the narrow intrinsic line width, the $21-\mathrm{cm}$ absorption systems are proposed to be used 
to directly measure the cosmic acceleration (Darling 2012; Yu et al. 2014), via the Sandage-Loeb (SL) effect (Sandage 1962; Loeb 1998). The main source of uncertainty of such proposed measurements lies in our poor understandings of H I clouds (Yu et al. 2014). New surveys are required to improve our understandings of spatial distribution and physical properties of HI clouds. This can be done by the Chinese new generation radio telescopes - Five-hundred-meter Aperture Spherical radio Telescope (FAST) (Li \& Pan 2016) and Tianlai 21-cm cosmology experiment (Tianlai) (Chen 2012). The singledish FAST, like Arecibo ${ }^{1}$, uses its gigantic single dish to achieve ultimate sensitivity, whereas Tianlai, designed like $\mathrm{CHIME}^{2}$, has ultra wide field of view (FoV) provided by its using its cylindrical reflectors and arrays of receivers to quickly scan the northern hemisphere of the sky as the Earth rotates.

Considering their respective design and observing strategies, we forecast their capabilities of blind searching of 21-cm absorption systems. In section 2 we show the sensitivity estimation and related factors for FAST and Tianlai. We present our forecast in section 3 and section 4 respectively. Conclusions are made in section 5 .

\section{SENSITIVITY}

To find possible 21-cm absorption systems, radio telescopes could be devised to scan the radio sources in NRAO VLA Sky Survey (NVSS) ${ }^{3}$. If we assume the distributions of radio sources and H I clouds are uncorrelated over the sky, the redshift distribution of the $21-\mathrm{cm}$ absorption systems $n_{\text {system }}$ would be

$$
n_{\text {system }}(z)=n_{\mathrm{HI}}^{*}(z) \int_{z}^{\infty} n_{R}\left(z^{\prime}\right) \mathrm{d} z^{\prime}
$$

where $n_{R}$ is the redshift distribution of radio sources over the sky (given by equation (26) of de Zotti et al. (2010)), and $n_{\mathrm{HI}}^{*}(z)$ is the number of occupation of H I clouds per any given line of sight. Note that, unlike $n_{R}$ and $n_{\text {system }}, n_{\mathrm{HI}}^{*}(z)$ is not an integration of volume so we do not need a volume filling factor in equation 1 . However, this spacial distribution of $\mathrm{HI}$ is poorly understood and is to be studied in upcoming surveys. Recent studies show that the number density of absorbers to be $\mathrm{d} n_{\mathrm{HI}} / \mathrm{d} z=n_{\mathrm{HI}}^{*}(z)=0.045 \pm 0.006$ per line of sight at low and medium redshift (Wolfe et al. 2005; Zwaan et al. 2007), so we apply this value in the following forcast. Equation 1 gives the H I cloud occupation regardless of observabilty, based on which we calculate the detections considering sensitivities.

Observation sensitivity depends on the background fluxes and foreground absorptions (current section), as well as telescope configurations and observation strategies (section 3,4).

NVSS contains 2 million sources (with declination $\delta>-40^{\circ}$, cover $82 \%$ of the sky) stronger than $2.5 \mathrm{mJy}$ at $\nu=1.4 \mathrm{GHz}$ (Condon et al. 1998) and their flux distribution $n_{R}(F)$ is given by Condon (1984). The observed flux is already lowered by the $21 \mathrm{~cm}$ absorption if there is any on the line of sight, so the actual signal to noise of the absorption line is higher. Due to the limited knowledge of absorption system properties we ignore this factor for now. Redshifted HI clouds absorb lower frequency bands where the radio sources are typically brighter by $\nu^{-0.7}$ (Condon et al. 1998), or $\left(1+z_{\mathrm{H} \mathrm{I}}\right)^{0.7}$. The error of the measurement $\Delta F$ is given by ${ }^{4}$

$$
\Delta F=T_{\mathrm{sys}} A_{\mathrm{eff}}^{-1} / \sqrt{n_{\mathrm{pol}} \Delta \nu \Delta t}
$$

where $T_{\text {sys }}$ and $A_{\text {eff }}$ are system temperature and effective receiving area, and $T_{\text {sys }} A_{\text {eff }}^{-1}$ is just the system equivalent flux density (SEFD). $n_{\mathrm{pol}}$ is the number of polarizations, $\Delta \nu$ is the line width and $\Delta t$ is the integration time. $\Delta \nu=\left(u_{\text {width }} / c\right) \nu_{\mathrm{obs}}$, where $u_{\text {width }}$ is the equivalent line width of the HI absorber. The properties of $21-\mathrm{cm}$ absorbers are primarily derived from followup studies of optical absorbers. The discovery rate from the cross correlation is a lower bound on the expect number of absorbers, since high column density systems in the CNM may systematically obscure potential background optical sources

\footnotetext{
1 https://www.naic.edu

2 http://chime.phas.ubc.ca

3 http://www.cv.nrao.edu/nvss/

4 https://science.nrao.edu/facilities/vla/docs/manuals/oss/performance/sensitivity
} 
(Yu et al. 2014). There are only 3 blind radio detections, and a survey may discover more systems which are optically obscured. They would likely be cold and at high column density. For sensitivity purposes, we treat all sources as $u_{\text {width }}=2 \mathrm{~km} \mathrm{~s}^{-1}$ (Wolfe et al. 1982, 2005). $\nu_{\text {obs }}$ is the observation frequency. For redshifted $21-\mathrm{cm}$ absorption systems, $\nu_{\mathrm{obs}}=1420 \mathrm{MHz} /\left(1+z_{\mathrm{HI}}\right)$. The integration time $\Delta t=\left(n_{\mathrm{obs}} \times 24 \mathrm{~h}\right) \tau$, where $n_{\mathrm{obs}}$ is the number of days an object is scanned, and $\tau=\lambda_{\text {obs }} / 2 \pi D \cos \delta$ is the fractional time the object transits the FoV $\left(\lambda_{\text {obs }} \ll D\right.$ or $\left.\delta \nrightarrow \pi / 2\right)$. For 21-cm absorption systems $\lambda_{\text {obs }}=21 \mathrm{~cm} \times\left(1+z_{\text {obs }}\right) . \Delta F$ is redshift- (frequency-) independent, as $\left(1+z_{\text {obs }}\right)$ terms from integration time and line width cancel out in the equation (2).

In the forecast we count $>10 \sigma$ detections, i.e., the absorption line depth is at least $10 \Delta F$. The optical depth of H I absorbers are also poorly understood. Recent discoveries of 21-cm absorption systems have about $r=20 \%$ fractional depth (Allison et al. 2015; Zwaan et al. 2015), and we apply this value. Thus, all systems with $r F>10 \Delta F$ are counted. Note that there is no confusion in the spectral domain - When the system temperature is not dominated by sources, the noise in each pixel is not affected by the number of sources. So only sources with absorbers contribute to the signal, and nothing contributes to the noise. Thus being confusion limited makes no difference.

\section{FAST ESTIMATION}

FAST has a primary dish of 500 meter in diameter. The effective antenna aperture of FAST is $300 \mathrm{~m}$, in diameter for any zenith angle up to $26^{\circ}$ and decreases to $200 \mathrm{~m}$ for a maximum zenith angle of $40^{\circ}$ (Li \& Pan 2016). The 19-beam feed-horn array at L band will be the primary survey instrument for FAST. For a single day it can scan total of $0.5^{\circ}$ in declination $\delta$. FAST survey strategy scans each object only once $\left(n_{\mathrm{obs}}=1\right)$, and with its high sensitivity, even faint sources are identified in a single day. We assume a one-month survey by FAST around the celestial equator for simplicity. FAST observes 1.02 to $1.42 \mathrm{GHz}$ (redshift $z_{\mathrm{H}}<0.39$ ), so objects on celestial equator have average transit time $\Delta t \simeq 12 \mathrm{sec}$. Taking $\Delta \nu \simeq 9 \mathrm{kHz}, n_{\mathrm{pol}}=2$ (dual polarized system), FAST has SEFD of $\sim 1 \mathrm{Jy}$, then we have $\Delta F \simeq 2.3 \mathrm{mJy}$. Taking into account the $\nu^{-0.7}$ flux boost at lower frequencies, $r F>10 \Delta F$ requires $F \gtrsim 0.11 \mathrm{Jy}$ on $1.4 \mathrm{GHz}$.

One-month-scan $\left(n_{\text {obs }}=30\right)$ around celestial equator by FAST will have $N_{R} \simeq 10^{4}$ sources whose $F \gtrsim 0.11 \mathrm{Jy}$ on $1.4 \mathrm{GHz}$. We further assume the independency between $n_{R}(F), n_{R}(z)$ and $n_{\mathrm{HI}}(z)$, from $n_{\text {system }}(z)$ by integrating equation (1), there will be $N_{\text {system }} \simeq 200 /$ month absorption systems found in redshift range $0<z<0.39$.

\section{TIANLAI ESTIMATION}

Tianlai is currently $30 \times 12 \mathrm{~m}$ area with SEFD $\simeq 300 \mathrm{Jy}$. Different from FAST, however, Tianlai scans all sources in the northern hemisphere of the sky everyday, and one needs longer periods integration to identify absorbers with fainter background sources. We forecast its one-year-survey capability of searching 21-cm absorption systems in the northern hemisphere.

Because Tianlai locates at latitude $\phi_{\text {site }}=+44^{\circ}$ and its cylinders are fixed, only objects at the zenith $\left(\delta=\phi_{\text {cite }}=44^{\circ}\right)$ fully utilize $A_{\text {eff }}$. On the other hand, higher declination objects have longer integration time per day. Thus, $\Delta F$ is a function of $\delta$,

$$
\Delta F(\delta)=\frac{T_{\text {sys }} A_{\text {eff }}^{-1}}{\cos \left(\delta-\phi_{\text {site }}\right)} \sqrt{\frac{\cos \delta}{n_{\text {pol }} \Delta \nu \Delta t(\delta=0)}},
$$

where $\Delta t(\delta=0) \simeq 1.17 \times 10^{5} \mathrm{sec}$ is the total integration time (1-year survey) for objects on celestial equator, for Tianlai frequency range 800 to $900 \mathrm{MHz}(0.58<z<0.78)$. The effective $N_{R}$ is given by

$$
N_{R}=\int_{0}^{\pi / 2} 2 \pi \cos \delta\left(\int_{10 \Delta F(\delta) / r}^{+\infty} n_{R}\left(F^{\prime}\right) \mathrm{d} F^{\prime}\right) \mathrm{d} \delta .
$$


Note that the inner flux integration converges as $F^{\prime} \rightarrow+\infty$ because $n_{R}(F) \sim F^{-2.5}$. Additionally, even if $\tau=\lambda_{\text {obs }} / 2 \pi D \cos \delta$ fails at $\delta \rightarrow \pi / 2$ (the north pole $\tau(\delta=\pi / 2)=1$ is always in FoV), the outer declination integration also converges as $\delta \rightarrow \pi / 2$, because the pole area is tiny and neglectable. For FAST, surveys at higher declinations would as well use equation $(3,4)$, however $\cos \left(\delta-\phi_{\text {site }}\right)$ term affecting SEFD should be neglected.

For Tianlai, equation (4) gives $N_{R} \simeq 1.3 \times 10^{4}$, and applying it to equation (1) again we get $N_{\text {system }} \simeq 80 /$ year absorption systems found in redshift range $0.58<z<0.78$.

\section{CONCLUSION}

We forecast the capability of FAST and Tianlai telescopes to search $21-\mathrm{cm}$ absorption systems. According to our assumptions, FAST is able to find $\simeq 200$ systems per month whereas TianLai can find $\simeq 80$ systems per year. Comparing the results between two quite different telescopes - high sensitivity FAST and large FoV Tianlai, we find the former is more efficient in looking for fainter radio sources $\left(n_{R}(F) \sim F^{-2.5}\right)$ for possible foreground absorptions. Future improvements in the sensitivity on Tianlai enable it more effectively looking for highest signal-to-noise systems over a wider sky.

Regarding the quantitative forecasts in each telescope, although we take into account many detailed aspects affecting the result, the major uncertainties are $n_{\mathrm{HI}}(z), u_{\text {width }}$ and $r$. These poorly understood parameters are conversely worth investigating from these proposed surveys. Modest real-time analysis changes could allow the survey of $21-\mathrm{cm}$ absorption systems. The data would need to be recorded at sufficient spectral resolution. Spatial computational costs are in principle unchanged, but there could be additional overhead costs for the larger resulting data sets. Systematic surveys of H I clouds over a cosmic scale enable us to measure the 3D density of these systems. For objects detected by FAST, one can also try to measure the $21 \mathrm{~cm}$ emission size. For Tianlai, one can try to measure the size from very-long-baseline interferometry (VLBI).

Acknowledgements This work was supported by the National Science Foundation of China (Grants No. 11573006, 11528306), the Ministry of Science and Technology National Basic Science program (project 973) under grant No. 2012CB821804. HRY acknowledges General Financial Grant No.2015M570884 and Special Financial Grant No. 2016T90009 from the China Postdoctoral Science Foundation. HRY and ULP acknowledge the support of the National Science and Engineering Research Council of Canada.

\section{References}

Allison, J. R., Sadler, E. M., Moss, V. A., et al. 2015, MNRAS, 453, 1249

Chen, X. 2012, International Journal of Modern Physics Conference Series, 12, 256

Condon, J. J. 1984, ApJ, 287, 461

Condon, J. J., Cotton, W. D., Greisen, E. W., et al. 1998, AJ, 115, 1693

Darling, J. 2012, ApJ, 761, L26

de Zotti, G., Massardi, M., Negrello, M., \& Wall, J. 2010, A\&A Rev., 18, 1

Li, D., \& Pan, Z. 2016, Radio Science, 51, 1060

Loeb, A. 1998, ApJ, 499, L111

Sandage, A. 1962, ApJ, 136, 319

Wolfe, A. M., Briggs, F. H., \& Davis, M. M. 1982, ApJ, 259, 495

Wolfe, A. M., Gawiser, E., \& Prochaska, J. X. 2005, ARA\&A, 43, 861

Yu, H.-R., Zhang, T.-J., \& Pen, U.-L. 2014, Physical Review Letters, 113, 041303

Zwaan, M. A., Liske, J., Péroux, C., et al. 2015, MNRAS, 453, 1268

Zwaan, M. A., van der Hulst, J. M., Briggs, F. H., Verheijen, M. A. W., \& Ryan-Weber, E. V. 2007, Astrophysics and Space Science Proceedings, 3, 501 